\title{
Phenotypic and genotypic detection of methicillin-resistant Staphylococcus aureus in hunting dogs in Maiduguri metropolitan, Borno State, Nigeria
}

\author{
Muhammad Mustapha, Yachilla Maryam Bukar-Kolo, Yaqub Ahmed Geidam and Isa Adamu Gulani \\ Department of Veterinary Medicine, Faculty of Veterinary Medicine, University of Maiduguri, PMB 1069 Maiduguri, \\ Borno State, Nigeria. \\ Corresponding author: Muhammad Mustapha, e-mail: tanimuzimbos@yahoo.co.uk, \\ YMB: yachillabukar@yahoo.com, YAG: yageidam@unimaid.edu.ng, IAG: isagulani@gmail.com \\ Received: 02-02-2016, Accepted: 13-04-2016, Published online: 24-05-2016
}

doi: 10.14202/vetworld.2016.501-506 How to cite this article: Mustapha M, Bukar-Kolo YM, Geidam YA, Gulani IA (2016) Phenotypic and genotypic detection of methicillin-resistant Staphylococcus aureus in hunting dogs in Maiduguri metropolitan, Borno State, Nigeria, Veterinary World, 9(5): 501-506.

\begin{abstract}
Aim: To determine the presence of MRSA in hunting dogs in Maiduguri metropolitan.

Materials and Methods: Phenotypic methods used includes microscopic technique, colony morphology study, catalasecoagulase tests, and the use of mannitol salt agar test, oxacillin resistance screening agar base, and antibiotic susceptibility testing methods. Genotypic approach was used for deoxyribonucleic acid extraction, and the presence of nuc and mecA gene was detected using polymerase chain reaction (PCR) techniques.

Results: Examination of 416 swab samples from nasal and perineal region of dogs revealed a total of $79.5 \%$ of S. aureus, where $62.5 \%$ of the isolates were MRSA. Molecular analysis revealed that 7 nuc genes specific for $S$. aureus from 20 presumptive MRSA assay were all mecA PCR negative. The isolates were sensitive to gentamicin and ciprofloxacin but proved resistant to cefoxitin and oxacillin.
\end{abstract}

Conclusion: High isolation rate of MRSA was found in hunting dogs. Significant level $(p<0.05)$ of MRSA was isolated in the nasal cavity of hunting dogs than its perineum. Only nuc genes were detected from the MRSA isolates.

Keywords: dogs, genotypic, methicillin, phenotypic, Staphylococcus aureus.

\section{Introduction}

Staphylococcal infections are of major importance in both Human and Veterinary Medicine. Staphylococcus aureus is a major resident or transient colonizer of the skin and the mucosa of humans and primates [1]. S. aureus is occasionally found on domestic animals, although other species of staphylococci predominate. S.aureus produces an extracellular thermostable nuclease, encoded by nuс gene [2].

$S$. aureus has characteristic ability to rapidly develop resistance to virtually any antibiotics coming into clinical use [3]. Resistance to methicillin that indicated resistance to all beta-lactam agents was first reported in 1961, which marked the appearance of methicillin-resistant $S$. aureus (MRSA) [4]. MRSA is becoming a major public health concern because companion animals often are in close physical contact (touching, petting, and licking) with their owners, exposing them to infection with pathogenic bacteria [5]. Dogs are usually colonized by MRSA strains from humans $[6,7]$.

Copyright: Mustapha, et al. Open Access. This article is distributed under the terms of the Creative Commons Attribution 4.0 International License (http://creativecommons.org/licenses/ by/4.0/), which permits unrestricted use, distribution, and reproduction in any medium, provided you give appropriate credit to the original author(s) and the source, provide a link to the Creative Commons license, and indicate if changes were made. The Creative Commons Public Domain Dedication waiver (http:// creativecommons.org/publicdomain/zero/1.0/) applies to the data made available in this article, unless otherwise stated.
When $S$. aureus gains entry into the host, it is able to cause a variety of infections from mild skin infection to life-threatening invasive infections such as brain abscesses, endocarditis, pericarditis, pneumonia, arthritis, osteomyelitis, urinary tract infection, and toxic shock syndrome [1]. The report above therefore, indicates that hunting dogs could be colonized by MRSA. Colonization of MRSA in dogs has been extensively studied in Europe and most parts of the world. However, in Nigeria, there is very little documented information on (MRSA) colonization of dogs.

There is the presence of MRSA in hunting dogs in Maiduguri Borno State, which is evident as a result of the presence of mecA polymerase chain reaction (PCR) negative $S$. aureus in the isolate. Therefore, the objective of this research is to determine the presence of MRSA, antibiotic sensitivity pattern of MRSA isolates, and the presence of nuc and mecA gene from MRSA isolates using phenotypic and genotypic techniques.

\section{Materials and Methods}

Ethical approval

This research was approved by the Faculty of Veterinary Medicine Ethics and Research Committee, University of Maiduguri, Borno State, Nigeria.

\section{Study area}

Maiduguri is the capital and the largest urban center of Borno State, North Eastern Nigeria (Figure-1). 


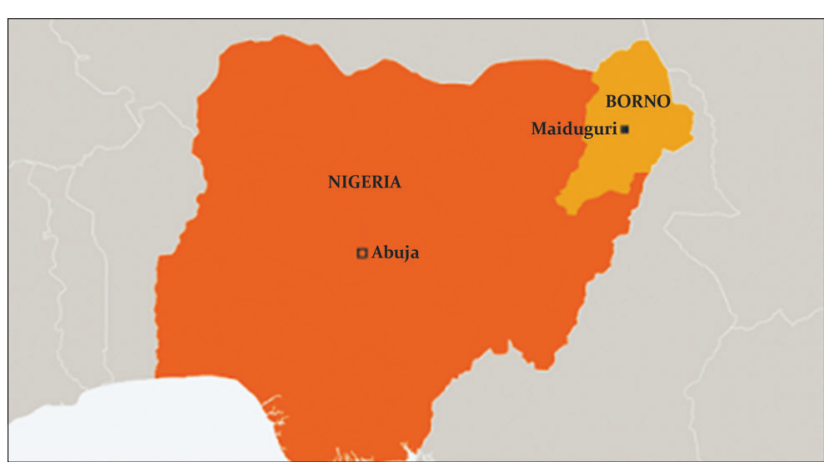

Figure-1: Map of Nigeria showing Maiduguri metropolitan in Borno State (study area).

The state lies between latitude $11^{\circ} 32^{\prime}$ North and $11^{\circ} 40^{\prime}$ North and latitude $13^{\circ} 20^{\prime}$ East and $13^{\circ} 25^{\prime}$ East between the Sudan Savanna and Sahel Savanna vegetation zones, characterized by short rainy season of 3-4 months (June-September) followed by a prolonged dry season of more than 8 months duration [8].

\section{Sample collection}

Total of 416 swab samples were collected from the four major hunting rendezvous in Maiduguri metropolitan. 211 and 205 swab samples from the nostril and perineum of the hunting dogs were analyzed. Cotton swab sticks were used to collect all the samples according to laboratory standard [9]. Each sample was labeled with an identification number and date of collection. The samples were kept on an ice pack and transported to the research laboratory, Department of Veterinary Medicine, Faculty of Veterinary Medicine, University of Maiduguri.

\section{Hunting dogs}

Dogs were sampled with owners consent. Cottontipped sterile swab (Everson Industries Limited, Nigeria) was inserted into one nostril of each dog where sample was taken from the nasal mucosa and immediately inserted into the aseptic tube. The tubes were labeled and dates of collection noted. Another sterile cotton-tipped swab is rolled on the perineal region and then immediately inserted back into its tube [9].

\section{Isolation and identification}

Enriched solid medium of 5\% blood agar (Sigma ${ }^{\circledR}$ Switzerland) was prepared according to the manufacturer's instruction and inoculated with the swabs [10]. Sterile wire loop was used to streak the inoculums to get discrete colonies. The inoculated plates were incubated aerobically at $37^{\circ} \mathrm{C}$ for $24 \mathrm{~h}$ and observed for yellowish-white colonies with smooth slightly raised surfaces. Some positive colonies have complete zones of hemolysis while others were non-hemolytic.

\section{Colony morphology}

Gram-stain of the collected samples was performed to identify staphylococci by their gram reaction. Samples that were cocci arranged in grape-like clusters were subjected to catalase and coagulase tests.
The positive isolates were streaked on mannitol salt agar (MSA, Oxoid) which is a selective medium for $S$. aureus, and plates were incubated under an aerobic condition at $37^{\circ} \mathrm{C}$ for $24 \mathrm{~h}$. The appearance of yellowish colonies on MSA was presumed to be $S$. aureus.

\section{Oxacillin resistance screening agar base (ORSAB)}

ORSAB is a medium for the screening of MRSA, the medium is nutritious, selective and contains peptones for the growth of microorganisms. The medium has high salt and lithium chloride concentration to suppress non-staphylococcal growth, with mannitol and aniline blue, for the detection of mannitol fermentation. The antibiotics contained in ORSAB selective supplement are oxacillin at $2 \mathrm{mg} / \mathrm{L}$ to inhibit methicillin-sensitive $S$. aureus, and polymyxin B for the suppression of other bacteria that are able to grow at such a high salt concentration,e.g. Proteus spp. typical colonies of MRSA are intense blue on a colorless background enabling the organism to be more easily identified in mixed culture than the pale yellow colonies seen on MSA.

\section{Antibiotic susceptibility testing}

The ATS of MRSA isolates was determined according to the method of Bauer-Kirby [11]. Using the commercially prepared disc (Oxoid, UK) with a known concentration of antibiotics, freshly sub-cultured MRSA and well-isolated colonies from ORSAB plates were emulsified in 3-4 $\mathrm{ml}$ of sterile normal saline. The turbidity of the suspension was adjusted to the turbidity of standard equivalent to 0.5 McFarland [12]. Mueller-Hinton agar medium was prepared, and a sterile cotton swab stick was dipped into the suspension. Excess fluid was removed by pressing and rotating the swab against the side of the tube above the suspension. The dried surface of the Mueller-Hinton agar was inoculated by streaking the swab evenly over the surface of the medium in three directions, rotating the plate approximately $60^{\circ}$ to ensure even distribution [9]. Five antimicrobial discs were dispensed into each inoculated plates and incubated at $35^{\circ} \mathrm{C}$ for $24 \mathrm{~h}$. Zone of inhibition were measured in millimeters ( $\mathrm{mm}$ ) using vernier caliper. The sizes of the zones of inhibition were interpreted according to CLSI [12] criteria. The following 10 antibiotics were tested FOX 30 ug, DA 2 ug, SXT 25 ug, CIP 5 ug, E 15 ug, KZ 30 ug, C 30 ug, CN $10 \mathrm{ug}$, TE $30 \mathrm{ug}$, and OX $1 \mathrm{~g}$ (Oxoid, UK). For the interpretation of susceptibility toward oxacillin disc, growth within the zone of inhibition was considered indicative of methicillin resistance. According to the classification criteria given by CLSI [12], a diameter of inhibition zones of $\leq 10,11-12$, and $\geq 13$ by $1 \mathrm{ug}$ of oxacillin is categorized as resistant (R), intermediate (I), or susceptible (S) to oxacillin accordingly. For cefoxitin disc, a diameter of inhibition zones of $\leq 24$ and $\geq 25 \mathrm{~mm}$ correspond to the class of staphylococci considered as resistance or susceptible for oxacillin, accordingly. There is no intermediate category 
of classification for staphylococci using the cefoxitin disc diffusion test [12].

\section{Genotypic characterization}

A total of 80 phenotypically detected MRSA from hunting dogs, 20 samples from this number were randomly selected for the genotypic analysis using PCR for the detection of $S$. aureus specific gene (nuc gene), and the mecA gene encoding the resistance as described by Perez-Roth et al. [13].

\section{Deoxyribonucleic acid (DNA) extraction}

A loop full MRSA isolates were scooped into $1.5 \mathrm{ml}$ tube that contained $400 \mu \mathrm{l}$ of lysis buffer and $4 \mu \mathrm{l}$ proteinase $\mathrm{k}$ and was vortexed (Fisher brand, Allied Fisher Scientific, USA), for 2 min to get a homogeneous mixture. This was followed by incubation at $55^{\circ} \mathrm{C}$ for $3 \mathrm{~h}$ in the thermocycler (Strata gene, USA). $400 \mu \mathrm{l}$ of phenol/chloroform (PC) was added to the tube and mixed gently for $1 \mathrm{~min}$. It was then spun in a microcentrifuge for $10 \mathrm{~min}$ at maximum speed $(10,000 \mathrm{rpm})$. The supernatant was transferred to another tube, and $400 \mu \mathrm{l}$ of PC was added, vortexed, and then it was spun for $10 \mathrm{~min}$ at 10,000 rpm. The supernatant was transferred to a new tube, $300 \mu$ of chloroform was added and vortexed, spun for $1 \mathrm{~min}$ at $10,000 \mathrm{rpm}$, and the supernatant was also transferred to another tube. $825 \mu \mathrm{l}$ of $100 \%$ ethanol and 25 $\mu l$ of sodium acetate were added and incubated in the freezer overnight.

The following day, the samples were centrifuged using high speed refrigerated centrifuge (Harvey Instruments Inc. USA) for $20 \mathrm{~min}$ and the supernatant was discarded. One $(1 \mathrm{ml})$ of $70 \%$ ethanol was added to the samples, mixed and centrifuged for $20 \mathrm{~min}$ at $13500 \mathrm{rpm}$; the supernatant was discarded and dry spun for $1 \mathrm{~min}$, and the residual ethanol was removed. The DNA pellets were allowed to dry at room temperature. The pellets were resuspended in $20 \mu \mathrm{l}$ of water. After the DNA extraction, $10 \mu 1$ of loading dye (bromophenol blue) was mixed with $5 \mu$ of the DNA pellets and pipetted into the wells of the gel. Finally, electrophoresis was carried out to determine the presence of DNA.

\section{PCR primers dilution}

Primers that corresponded to $n u c$ gene specific for $S$. aureus and mecA gene were obtained from Integrated DNA Technologies, USA. The primers were resuspended in sterile distilled water, and the diluted primers were stored at $-20^{\circ} \mathrm{C}$.

\section{Detection of nuc and mecA gene by PCR}

The PCR amplification was done according to Perez-Roth et al. [13]. Using the following primers that will detect the nuc gene in S. aureus and mecA gene with the amplicon size of $276 \mathrm{bp}$ and $533 \mathrm{bp}$; The nuc primers were 5'-GCG ATT GAT GGT GAT ACG GTT-3' and 5'- AGC CAA GCC TTG ACG AAC TAA AGC-3', whereas the mecA primers were 5'-AAA ATC GAT GGT AAA GGT TGG C-3' and
5'-AGT TCT GCA GTA CCG GAT TTG C-3'. The PCR amplification mixture consisted of $10 \mu 1$ of PCR premix, $2 \mu 1$ DNA templates, $2 \mu 1$ primers, and $16 \mu 1$ of distilled water. A total of 40 cycles were used to amplify $533 \mathrm{bp}$ of $m e c A$ gene and $276 \mathrm{bp}$ of $n u c$ gene specific for $S$. aureus. DNA pre-denaturation occurs at $94^{\circ} \mathrm{C}$ for $5 \mathrm{~min}$ and DNA denaturation at $30 \mathrm{~s}$ to $1 \mathrm{~min}$ primers annealing at $55^{\circ} \mathrm{C}$ for $30 \mathrm{~s}$. Approximately $5^{\circ} \mathrm{C}$ below primers temperature, extension of the two strands at $72^{\circ} \mathrm{C}$ for $60 \mathrm{~s}$ and a final extension at $72^{\circ} \mathrm{C}$ for $4 \mathrm{~min} .10 \mu \mathrm{l}$ each of the PCR products for mecA and nuc gene were analyzed separately on $2 \%$ agarose gel (Biogene, UK). Electrophoresis was performed in TBE buffer at $180 \mathrm{~V}$ for $1 \mathrm{~h}$, and the gel was subsequently stained with $3 \mu 1$ of ethidium bromide (Sigma, UK). DNA bands were visualized using UV-light with the camera (Gel Doc 2000, Bio-Rad) and photographed.

\section{Data analysis}

Fisher's exact test (Graph pad ${ }^{\circledR}$ Software Inc.) was used to determine the probability and significance of MRSA detection from the skin, perineal region, and nasal cavity of hunting dogs. The values were considered significant $(\mathrm{p}<0.05)$.

\section{Results}

Total of 416 swab samples from the nostril and perineal region of hunting dogs of $S$. aureus revealed 128 (79.5\%) positive isolates. Microscopic examination of Gram-stained colonies showed Gram-positive cocci arranged in irregular grape-like clusters, some appearing in single, whereas others in pairs, short chain, or tetrads. Results of catalase and coagulase positive isolates are 141 (87.6) and 128 (79.5\%), respectively. Table-1 shows results of Gram-staining, biochemical test and ORSAB screening of staphylococcal isolates in hunting dogs in Maiduguri metropolitan, Borno State. The values of $S$. aureus isolated from the nostril and perineum of hunting dogs were $78(36.9 \%)$ and $50(24.4 \%)$ respectively. The values of MRSA isolated from the nostril and perineum of hunting dogs were $50(23.7 \%)$ and $30(14.7 \%)$, respectively (Table-2). The result of antimicrobial susceptibility

Table-1: Gram-staining, biochemical test and ORSAB screening of $S$. aureus isolates in hunting dogs in Maiduguri metropolitan, Borno State.

\begin{tabular}{lccc}
\hline Test & $\begin{array}{c}\text { Result of } \\
\text { positive } \\
\text { isolates (\%) }\end{array}$ & $\begin{array}{c}\text { Result of } \\
\text { negative } \\
\text { isolates (\%) }\end{array}$ & Total \\
\hline Gram reaction & $161\left(38.7^{\mathrm{a}}\right)$ & $255\left(61.3^{\mathrm{b}}\right)$ & 416 \\
Catalase test & $141\left(87.6^{\mathrm{c}}\right)$ & $20\left(12.4^{\mathrm{d}}\right)$ & 161 \\
Coagulase test & $128(79.5)$ & $33\left(20.5^{\mathrm{y}}\right)$ & 161 \\
ORSAB screening & $80\left(62.5^{\mathrm{k}}\right)$ & $48\left(37.5^{\mathrm{y}}\right)$ & 128 \\
\hline
\end{tabular}

Figures in brackets are percentage occurrence of staphylococcal isolate species. Values denoted by different superscripts for a given parameter are significantly different $(p<0.05)$. ORSAB $=$ Oxacillin resistance screening agar base, $S$. aureus=Staphylococcus aureus 
Available at www.veterinaryworld.org/Vol.9/May-2016/12.pdf

Table-2: Percentage distribution of $S$. aureus and MRSA isolated from hunting dogs in Maiduguri metropolitan.

\begin{tabular}{llcccr}
\hline Source & Site & S. aureus (-ve) & S. aureus (+ve) & MRSA (+ve) & Number of samples \\
\hline Dog & Nasal & 133 & $78(36.9)$ & $50(23.7)$ & 211 \\
& Perineum & 155 & $50(24.4)$ & $30(14.7)$ & 205 \\
& Total & 288 & $128(30.8)$ & $80(19.2)$ & 416 \\
\hline
\end{tabular}

MRSA=Methicillin-resistance Staphylococcus aureus, S. aureus=Staphylococcus aureus

Table-3: Antibiotic susceptibility pattern of MRSA isolated from hunting dogs in Maiduguri metropolitan, Borno State.

\begin{tabular}{lccc}
\hline \multicolumn{2}{c}{ Susceptibility pattern } \\
\hline Antibiotics & $\begin{array}{l}\text { Resistant number } \\
\text { of isolates (\%) }\end{array}$ & $\begin{array}{l}\text { Intermediate number } \\
\text { of isolates (\%) }\end{array}$ & $\begin{array}{l}\text { Susceptible number } \\
\text { of isolates (\%) }\end{array}$ \\
\hline Cefoxitin & $97(99.0)$ & $0(0.0)$ & $1(1.0)$ \\
Cefazolin & $69(70.0)$ & $6(6.1)$ & $23(23.5)$ \\
Chloramphenicol & $8(8.2)$ & $4(4.1)$ & $86(87.8)$ \\
Ciprofloxacin & $3(3.1)$ & $6(6.1)$ & $89(90.8)$ \\
Clindamycin & $15(15.3)$ & $5(5.1)$ & $78(79.6)$ \\
Erythromycin & $14(14.3)$ & $4(4.1)$ & $80(81.6)$ \\
Gentamicin & $1(1.0)$ & $1(1.0)$ & $96(98.9)$ \\
Oxacillin & $87(88.8)$ & $1(1.0)$ & $10(10.2)$ \\
Tetracycline & $72(73.4)$ & $4(4.1)$ & $22(22.4)$ \\
Sulfa/Trimethoprim & $7(7.1)$ & $5(5.1)$ & $86(87.8)$ \\
\hline
\end{tabular}

MRSA=Methicillin-resistance Staphylococcus aureus

Table-4: Multidrug resistance profile of MRSA isolated from hunting dogs in Maiduguri metropolitan.

\section{Number of drugs resisted (\%)}

$0(0.00)$

$1(2.04)$

$2(6.12)$

$3(34.7)$

$4(35.7)$

$\geq 5$ (21.4)

MRSA $=$ Methicillin-resistance Staphylococcus aureus

test indicated that the isolates were highly resistant to FOX (99\%), OX (88.7\%), TE (73.4\%), and $\mathrm{KZ}(70.0 \%)$, while they were highly susceptible to CN (98\%), CIP (90.8\%), C (87.6\%), SXT (87.8\%), E $(81.6 \%)$, and DA(79.6\%) as presented in Table-3. Multidrug Resistance (MDR) Profile of MRSA isolated from hunting dogs in Maiduguri Metropolitan is shown in Table-4. 20 presumptive MRSA isolates assayed 7 bands showed evidence expression of nuc gene specific for $S$. aureus with a molecular weight of 276 bp which is presented in Figure-2. This confirms the assumption based on the phenotypic detection that some of the strains were $S$. aureus. The result of PCR based on targeted mecA gene revealed that none of the isolates possessed $m e c A$ gene as represented in Figure-3.

\section{Discussion}

Investigations on phenotypic detection of MRSA in hunting dogs in Maiduguri Metropolitan revealed higher isolation rates $(19.2 \%)$ of these pathogens in dogs compared to the findings of Loeffler et al. [14], Kottler et al. [15], and Chah et al. [16] who, respectively, recorded $9.0 \%, 3.3 \%$, and $12.8 \%$ of MRSA in dogs in the UK, the US, and Nigeria.

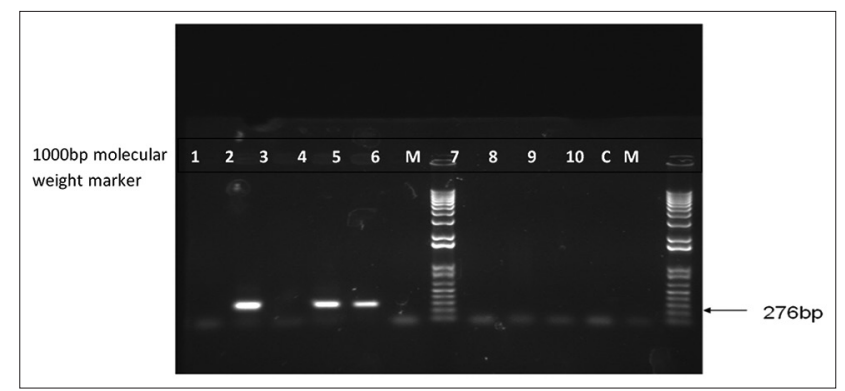

Figure-2: Agarose gel electrophoresis of polymerase chain reaction of methicillin-resistance Staphylococcus aureus isolate. Lanes 2, 4, and 5 are positive for nuc gene as indicated by 276 bp.Lane 1, 3, 6, 7, 8, 9, and 10 are negative samples. Lane $\mathrm{M}$ is the molecular weightmarker. Lane $\mathrm{C}$ is the negative control.

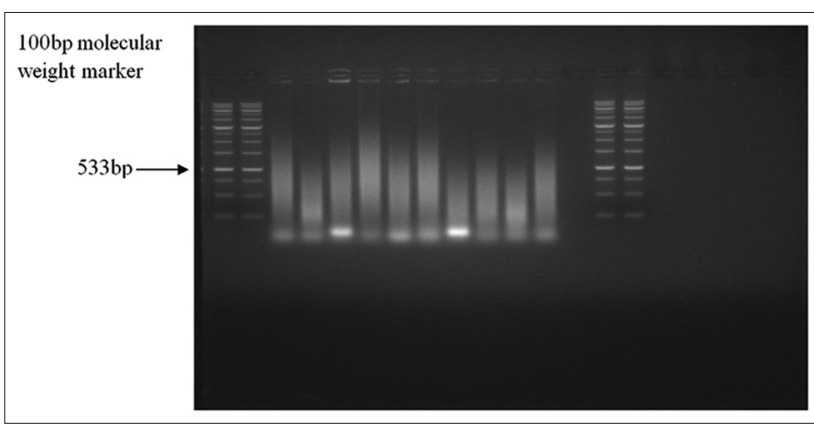

Figure-3: Agarose gel electrophoresis pattern of polymerase chain reaction of methicillin-resistance Staphylococcus aureus isolate. Lane M: Molecular weight marker. Lane 1-10: Negative for mecA. Lane C: Negative control.

This may probably be attributed to variation in the breeds of dogs used in this finding, indiscriminate antibiotic therapy, harsher environmental challenges commonly encountered during hunting and could also be associated to starvation which results in stress that affects dog's immunity. Kutdang et al.[17] supported 
the assertion above from his finding that hunting dogs are more exposed to the outside environment and hence stood the chance of contracting more infections than household dogs. Floras et al. [18] further augmented the findings of this study with a report of an increasingly higher detection rate of MRSA in dogs in Canada.

The distribution of $S$. aureus and MRSA recorded from the nasal cavity of hunting dogs was significantly different (higher) than the values recorded from the perineal region of dogs. These suggest that higher possibilities of contamination rate of dogs are through the nostril. Contaminations can also result through scavenging of death carcasses exposed to antibiotics from backyard poultry operations. These tallies, with the findings of Rich and Roberts [19] and Khanna et al. [20] who reported that MRSA colonization in animals, are better detected through nasal sampling. Rich and Roberts [19] further confirmed this through a record of a single case of MRSA detection from nasal swab samples of 255 dogs and not from the throat and skin of the same animals.

Antimicrobial susceptibility pattern of the MRSA isolates has also been investigated indicating a high level of resistance to FOX and OX. This finding, therefore, implies that FOX based assays are particularly important for low-level OX resistant MRSA detection [21]. Phenotypic antimicrobial susceptibility test needs particular care when using $\mathrm{OX}$ as a test substrate because of heterogeneous in vitro expression of Methicillin-resistance (hetero-resistance) in nearly all of currently disseminated MRSA clonal lineages. Heteroresistance can be either detected using high inocula as recommended by the Clinical Laboratory Standard Institutes, or by use of FOX disks since this antibiotic is less affected by heterogeneous expression [12].

The MRSA isolates were highly susceptible to CIP and CN. CIP, a member of the fluoroquinolones that are newer drugs with mode of action on DNA inhibition and are relatively expensive and less available for abuses [22]. In addition; $\mathrm{CN}$, an aminoglycoside, also showed high activity against MRSA, which may be as a result of the complexity of the aminoglycoside and the route of administration [22].

It was concluded that 59 out of 80 isolates of MRSA were multidrug resistant (MDR) in this study. This finding was supported by Abeer et al. [23], who reported that 14 out of 51 MRSA isolates were MDR. Methicillin-resistant coagulase negative staphylococci (MRCoNS) from healthy dogs in Nsukka, Nigeria, isolated revealed that 13 out of 109 isolates of MRCoNS were MDR [16].

The PCR analysis did not reveal any mecA positive samples, and this might indicate the presence of $m e c A$ PCR negative MRSA isolates in Maiduguri. A previous study by Garcia-Alvarez et al. [24] had reported a novel allele of the $m e c A$ gene encoding an alternative penicillin binding protein that mediates methicillin resistance among bovine $S$. aureus isolates, and humans in the UK, Denmark, and Germany that were Methicillin-resistant but mecA PCR negative.

These novel alleles of the $m e c A$ gene $\left(m e c A_{\mathrm{LGA}_{251}}\right)$ have $70 \%$ nucleotide identity to the archetypal mec $A$ gene. Moreover, the findings highlight the possibility that additional mecA allele is in circulation in the environment, and therefore, could be acquired by $S$. aureus and leads to the emergence of new MRSA strain. However, antimicrobial susceptibility testing and other routine culture will identify $S$. aureus isolates encoding $m e c A_{\mathrm{LGA251}}$ as methicillin-resistant [24]. Furthermore, Kriegeskorte et al. [25] also found MRSA isolates with novel genetic homolog among human in a study of human MRSA isolates in Germany.

The results of the PCR analysis in the current study which revealed nuc genes at 276 bp which tallied with the findings recorded by Merlino et al. [26], Saiful et al. [27], and Szczepanik et al. [28], who used modified PCR analysis (multiplex PCR) for the detection of mecA and nuc genes in multidrug resistance and non-multidrug resistance MRSA.

\section{Conclusion}

Conclusively, MRSA was phenotypically detected with significant $(p<0.05)$ isolation rate in the hunting dogs. Microbiological and PCR results confirm the presence of MRSA in hunting dogs in Maiduguri Metropolitan, Borno State, Nigeria. Higher percentages $(50.0 \%)$ of MRSA were detected from the nasal cavities of dogs than the perineal region (30.0\%).

\section{Author's Contributions}

This study was conceived by MM, YMB, and YAG. MM collected the data during field work. The data were compiled by MM, analyzed by MM and IAG. Write up by MM. All authors read and approved the final manuscript.

\section{Acknowledgments}

My sincere appreciation goes to pet owners for permission to collect samples, DNA laboratory Kaduna, Nigeria, for molecular analysis and Dr. M.S. Auwal for objective criticism. The research is self-sponsored.

\section{Competing Interests}

The authors declare that they have no competing interests.

\section{References}

1. Pantosti, A. (2012) Methicillin-resistant Staphylococcus aureus associated with animal and its relevance to human. Microbiol, 3: 137.

2. Zhang, K., Sparkling, J., Chow, B.L., Elsayed, S., Hussain, Z. and Church, D.C. (2004) New quadriplex PCR assay for detection of methicillin and mupirocin resistance and simultaneous discrimination of Staphylococcus aureus from coagulase-negative Staphylococci. J. Clin.Microbiol., 42(11): 4947-4955. 
3. Pantosti,A., Sanchini,A. and Monaco,M.(2007)Mechanisms of antibiotic resistance in Staphylococcusaureus. FutureMicrobiol., 2(3): 323-334.

4. Molton, J.S., Tambyan, P.A. and Ang, B.S.P. (2013) The global spread of healthcare associated multidrug-resistant bacteria: A perspective from Asia. Clin. Infect. Dis., 56(3): 1310-1318.

5. Guardabassi, L., Loeber, M.E. and Jacobson, A. (2004) Transmission of multiple antimicrobial-resistant Staphylococcus intermedius between dogs affected by deep pyoderma and their owners. Vet. Microbiol., 98:23-27.

6. Loeffler, A. and Lloyd, D.H. (2010) Companion animals: A reservoir for methicillin-resistant Staphylococcus aureus in the community? Epidemiol. Infect., 138(5):595-605.

7. Lin, Y., Barker, E., Kislow, J., Kaldhone, P., Stemper, M.E., Pantrangi, M., Moore, F.M., Hall, M., Fritsche, T.R., Novicki, T., Foley, S.L. and Shukla, S.K. (2011) Evidence of multiple virulence subtypes in nosocomial and community-associated MRSA genotypes in companion animals from the upper Midwestern and northeastern United States.Clin. Med. Res., 9(1):7-16.

8. Borno State Ministry of Land and Survey, (BMLS). (2016) Annual Report 15-58.

9. Cheesbrough, M. (2010) District Laboratory Practice in Tropical Countries. $2^{\text {nd }}$ ed. Cambridge University Press, Cambridge, UK. p45.

10. Vincze, A., Stamm, I., Kopp, P.A., Hermes, T., Adlhoh, C., Semmler, T., Wieler, LH., Lubke-Becker, A. and Walter, B. (2014) Alarming proportion on Methicillin-resistant Staphylococcus aureus (MRSA) in wound samples from companion animals, Germany 2010-2012. PLoSOne, 9(1): e85656.

11. Bauer, A.W., Kirby, W.M.M., Sherris, J.C. and Turck, M. (1966) Antibiotic susceptibility testing by standardized single disk method. Am. J. Clin. Pathol., 45: 493-496.

12. CLSI. (2011) Performance Standards for Antimicrobial SusceptibilityTesting; Twenty- First Information Supplement. M100-S20.Vol.30. Clinical Laboratory Standard Institute, Wayne, PA, p157-165.

13. Perez-Roth, E., Claverie-Martin, F., Villar, J. and MendezAlvarez, S. (2001) Multiplex PCR for simultaneous identification of Staphylococcus aureus and detection of methicillin and mupirocin resistance. J. Clin. Microbiol.,39: 4037-4041.

14. Loeffler, A., Boag, A.K., Sung, J., Lindsay, J.A., Guardabassi, L., Dalsgaard, A., Smith, H., Stevens, K.B. and Lloyd, D.H. (2005) Prevalence of methicillin-resistant Staphylococcus aureus among staff and pets in a small animal referral hospital in the UK. J. Antimicrob. Chemother., 56(4): 692-697.

15. Kottler, S., Middleton, J.R., Perry, J., Weese, J.S. and Cohn, L.A. (2010) Prevalence of Staphylococcusaureus and methicillin-resistant Staphylococcusaureus carriage in three populations. J. Vet. Int. Med. 24(1):132-139.

16. Chah, K.F., Soaz, E.G., Nwanta, J.A., Asadu, B., Agbo, I.F., Lozano, C., Zarazaga, M. and Torres, C. (2014) Methicillinresistant coagulase negative Staphylococci from healthy dogs in Nsukka, Nigeria. Braz. J. Microbiol., 45(1): 215-220.

17. Kutdang, E.T., Bukbuk, D.N. and Ajayi, J.A.A. (2010) Theprevalence of intestinalhelminthes of dogs (Canisfamillaris) in Jos, Plateau State, Nigeria. Researcher, 2(8):51-56.

18. Floras, A., Lawn, K., Slavic, D., Golding, G.R., Mulvey, M.R. and Weese, J.S. (2010) Sequence type 398 meticillin-resistant Staphylococcus aureus infection and colonisation in dogs.Vet. Rec., 166(26):826-827.

19. Rich, M. and Roberts, L. (2004) Methicillin-resistant Staphylococcus aureusisolates from companion animals. Vet. Rec., 154(10):310.

20. Khanna, T., Friendship, R., Dewey, C. and Weese, J.S. (2008) Methicillin resistant Staphylococcus aureus colonization in pigs and pig farmers. Vet.Microbiol., 128(3-4):298-303.

21. Witte, W., Pasemann, B. and Cuny, C. (2007) Detection of low-level oxacillin resistance in mecA positive Staphylococcus aureus.Clin. Microbiol. Infect., 13(4): 408-412.

22. Onanuga, A., Oyi, A.R. and Onaolapa, A.J. (2005) Prevalence and susceptibility pattern of Methicillin resistant Staphylococcus aureus isolates amongst healthy women in Zaria,Nigeria. Afr. J. Biotechnol., 4(11):1321-1324.

23. Abeer, A.R., Mohamed, S.S. and Amal, S.O. (2007) Detection of methicillin/oxacillin resistant Staphylococcus aureus isolated from clinical Hospital in Cairo using mecA/ nuc gene and antibiotic susceptibility profile. Int.J. Agric. Biol., 9(6):800-806:

24. Garcia-Alvarez, L., Holden, M.T., Lindsay, H., Webb, C.R., Brown, D.F. and Curran, M.D. (2011) Methicillin-resistant Staphylococcus aureus with a novel mecA homologue in human and bovine population in the UK and Denmark: A descriptive study. Lancet Infect.Dis., 11:595-603.

25. Kriegeskorte, A., Ballhausen, B., Idelevich, E.A., Kock, R., Friedrich, A.W. and Karch, H. (2012) Human methicillin-resistant Staphylococcus aureus isolates with novel genetic homolog, Germany. Emerg.Infect. Dis., 18(6):1016-1018.

26. Merlino, J., Watson, J., Rose, B., Beard-Pegler, M., Gottlieb, T., Bradbury, R. and Harbour, C. (2002) Detection and expression of methicillin/oxacillin resistance in multidrug-resistant and non-multidrug-resistant Staphylococcus aureus in Central Sydney, Australia. J. Antimicrob. Chemother., 49: 793-801.

27. Saiful, A.J., Mastura, M., Zarizal, S., Mazurah, M.I., Shuhaimi, M. and Ali, A.M. (2006) Detection of methicillin-resistant Staphylococcus aureus using mecA/nuc genes and antibiotic susceptibility profile of Malaysian clinical isolates.World J. Microbiol. Biotechnol., 22:1289-1294.

28. Szczepanik, A., Koziol-Montewka, M., Al-Doori, Z., Morrison, D. and Kaczor, D. (2007) Spread of a single multi-resistant methicillin-resistant Staphylococcus aureus clone carring a variant of staphylococcal cassette chromosome mecType III isolated in a university hospital. Eur. J. Clin. Microbiol. Infect. Dis., 26: 29-35. 